\title{
ON RINGS WHOSE MODULES HAVE NONZERO HOMOMORPHISMS TO NONZERO SUBMODULES
}

\author{
Y. TOlOOEI AND M. R. Vedadi
}

\begin{abstract}
We carry out a study of rings $R$ for which $\operatorname{Hom}_{R}(M, N) \neq 0$ for all nonzero $N \leq M_{R}$. Such rings are called retractable. For a retractable ring, Artinian condition and having Krull dimension are equivalent. Furthermore, a right Artinian ring in which prime ideals commute is precisely a right Noetherian retractable ring. Retractable rings are characterized in several ways. They form a class of rings that properly lies between the class of pseudo-Frobenius rings, and the class of max divisible rings for which the converse of Schur's lemma holds. For several types of rings, including commutative rings, retractability is equivalent to semi-Artinian condition. We show that a Köthe ring $R$ is an Artinian principal ideal ring if and only if it is a certain retractable ring, and determine when $R$ is retractable.
\end{abstract}

2010 Mathematics Subject Classification: Primary: 16D10; Secondary: 16E50, 16L30, 16A55.

Key words: CPF rings, max ring, regular ring, retractable, semi-Artinian.

\section{Introduction}

Throughout this paper rings will have unit elements and modules will be right unitary. Following [12], an $R$-module $M$ is called retractable if $\operatorname{Hom}_{R}(M, N) \neq 0$ for all nonzero submodules $N$ of $M$. Semisimple modules and fully idempotent modules $[\mathbf{2 1}]$ are clearly retractable, and more generally self-projective modules with zero radical and essentially compressible modules are known to enjoy this property; see [5, 3.4] and [19, Theorem 3.1]. Retractable modules have appeared in different situations. For example, in the study of nonsingular modules satisfying one of the properties: CS, continuous, quasi continuous or having a Baer endomorphism ring [16, Theorem 22]. They have also been applied in the study of prime $M$-ideals that correspond to the isomorphism classes of indecomposable $M$-injective modules in $\sigma[M]$ [3, Theorems 2.10 and 6.7] and in the characterization of endomorphism rings of quasi-injective envelopes of polyform modules [5, 5.19]; see also, [9, Theorem 2.6], and [24, Section 2]. In [21], it is shown that the commutative rings over which every module is fully idempotent are exactly the semisimple rings. 
Rings with all finitely generated modules retractable are characterized in $[\mathbf{8}]$, and finitely generated retractable modules over right FBN rings are characterized in [18] where the term slightly compressible is used for retractable.

In the present work, we shall consider retractable rings which are rings with all nonzero module retractable. Recall from $[\mathbf{7}], R$ is a right CPF ring if for all proper ideals $I$ of $R$, any faithful $R / I$-module is a generator in Mod- $R / I$. Artinian principal ideal rings are CPF $[\mathbf{2 3}, 56.9(\mathrm{c})]$. In Proposition 2.4 we show that the class of retractable rings properly lies between the class of right CPF rings and the class of divisible right max rings which are "CS" in the sense of Hirano and Park [11]. These are rings for which the converse of Schur's Lemma holds; see also [10]. Some equivalent conditions for a ring to be retractable are given in Theorem 2.2, where it is shown that retractable rings are precisely rings over which all torsion theories are hereditary. Over a retractable ring, a module is Artinian if and only if it is Noetherian and its second singular submodule is Artinian (Proposition 2.10). Retractable rings with Krull dimension and reduced retractable rings are characterized in Theorems 3.6 and 3.2. More generally, retractable rings $R$ such that $R / \mathrm{J}(R)$ is reduced are shown to be left semi-Artinian, and they are precisely semi-Artinian if in addition $\mathrm{J}(R) \subseteq \operatorname{Cent}(R)$ (Theorem 3.4 and Corollary 3.5). A result of Köthe states that over an Artinian principal ideal ring $R$ every right (left) $R$-module is a direct sum of cyclic right (left) $R$-modules (i.e. $R$ is a Köthe ring) [13]. We investigate the converse of the Köthe theorem and as an application of our results, we show that a Köthe ring $R$ is an Artinian principal ideal ring if and only if it is a retractable ring such that for any ring decomposition $\operatorname{Mat}_{n}(S) \times T \simeq R$ with local $S$, the ring $S$ is Köthe (Theorem 3.10). The retractability of Köthe rings are then determined. Any unexplained terminology, and all the basic results on rings and modules that are used in the sequel can be found in $[\mathbf{2}],[\mathbf{5}]$ and $[\mathbf{1 4}]$.

\section{Retractability of modules}

In this section we investigate the class of retractable rings in Theorem 2.2 and Propositions 2.4, 2.6 and study modules over retractable rings. A class $\mathcal{C}$ of $R$-modules is called retractable if $X_{R}$ is retractable for all $X \in \mathcal{C}$. An $R$-module $M$ is called essentially retractable if $\operatorname{Hom}_{R}(M, N) \neq 0$ for all essential submodules $N$ of $M$; see [22] for more information about essentially retractable modules. For an $R$-module $M_{R}$, the injective hull of $M$ is denoted by $\mathrm{E}\left(M_{R}\right)$ or simply $\mathrm{E}(M)$. 
Lemma 2.1. The following statements are equivalent for a nonzero $R$-module $M$.

(i) $M_{R}$ is essentially retractable.

(ii) There exists a nonzero $f \in \operatorname{Hom}_{R}(M, \mathrm{E}(M))$ such that $f(M)$ is an essentially retractable $R$-module.

Proof: (i) $\Rightarrow$ (ii). This is clear.

(ii) $\Rightarrow$ (i). Suppose that (ii) holds. Let $K$ be an essential submodule in $M_{R}$. Then $K$ is essential in $\mathrm{E}(M)$, and hence $K \cap f(M)$ is essential in $f(M)$. Thus there exists a nonzero homomorphism from $f(M)$ to $K \cap f(M)$. It follows that $\operatorname{Hom}_{R}(M, K) \neq 0$.

Theorem 2.2. For a ring $R$, the following statements are equivalent.

(i) $R$ is a retractable ring.

(ii) Every nonzero $R$-module is essentially retractable.

(iii) Every essential extension of a cyclic $R$-module is essentially retractable.

(iv) $\operatorname{Hom}_{R}(M, X)=0 \Leftrightarrow \operatorname{Hom}_{R}(M, \mathrm{E}(X))=0$ for all $R$-modules $M$ and $X$.

(v) All torsion theories on $R$ are hereditary.

Proof: (i) $\Rightarrow$ (ii) and (ii) $\Rightarrow$ (iii) are clear.

(iii) $\Rightarrow$ (ii). Note that if $0 \neq m \in M_{R}$, then $m R$ essentially embeds in a suitable factor of $M_{R}$ [14, Proposition 6.18].

(ii) $\Rightarrow$ (iv). If $\operatorname{Hom}_{R}(M, \mathrm{E}(X))$ is nonzero, then similar to the proof of Lemma 2.1, we have $\operatorname{Hom}_{R}(M, X) \neq 0$. The converse is clear.

(iv) $\Rightarrow(\mathrm{v})$. Let $(\mathcal{T}, \mathcal{F})$ be a torsion theory on $R, N \leq M_{R} \in \mathcal{T}$ and $X \in \mathcal{F}$. Then $\operatorname{Hom}_{R}(M, X)=0$, hence $\operatorname{Hom}_{R}(M, \mathrm{E}(X))=0$ by (iv). It follows that $\operatorname{Hom}_{R}(N, X)=0$, proving that $N_{R} \in \mathcal{T}$.

(v) $\Rightarrow$ (i). Let $N \leq M_{R}$ and $(\mathcal{T}, \mathcal{F})$ be a torsion theory generated by $M_{R}$. By (v) $N_{R} \in \mathcal{T}$ and hence $\operatorname{Hom}_{R}(M, N) \neq 0$.

In the following we collect more properties of modules over retractable rings. A module $M_{R}$ is called divisible if $M c=M$ for any right regular

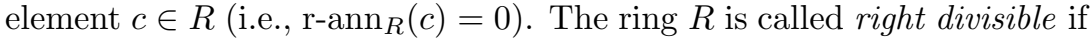
the module $R_{R}$ is divisible. It is well known that injective modules are divisible. If $M$ is an $R$-module such that $(M / N)_{R}$ and $N_{R}$ are divisible for some $N \leq M_{R}$, then it is easily seen that $M_{R}$ is divisible. 
Proposition 2.3. Let $R$ be a retractable ring and let $M$ and $N$ be nonzero $R$-modules.

(i) If $M I^{n}=0$ for some ideal $I$ of $R$ and $n \geq 1$, then $M c=M$ for every $c \in R$ which is right regular modulo $I$.

(ii) $\mathrm{J}(M) \neq M$.

(iii) If $M / \mathrm{J}(M)$ is a semisimple $R$-module and $\operatorname{Hom}_{R}(M, N) \neq 0$, then $\operatorname{Hom}_{R}(N / \mathrm{J}(N), M / \mathrm{J}(M)) \neq 0$.

(iv) The module $M_{R}$ is nonsingular if and only if every nonzero submodule of $M_{R}$ contains a nonzero injective projective submodule if and only if every nonzero submodule of $M_{R}$ contains a nonzero projective submodule.

(v) If $M_{R}$ is nonsingular, then $\mathrm{J}(M)=0$.

Proof: (i) We first show that any nonzero $R$-module is divisible. Let $U_{R}$ be nonzero. Then $E=\mathrm{E}(U)$ is a retractable $R$-module by our assumption. So there is a proper submodule $K$ of $E$ such that $U_{R}$ contains a submodule isomorphic to $E / K$. It follows that $U_{R}$ contains a nonzero divisible submodule. Consequently, if $N=\sum\left\{K \leq M_{R} \mid K\right.$ is divisible $\}$ for a given nonzero $R$-module $M_{R}$, then $N$ is a nonzero divisible submodule of $M_{R}$. If $M / N$ is nonzero, then it contains a nonzero divisible submodule $A / N$. It is easy to verify that $A_{R}$ is also divisible and so it lies in $N$, a contradiction. Thus $M=N$ and $M_{R}$ is divisible, as desired. Now let $M_{R}$ be nonzero and $M I^{n}=0$ for some ideal $I$ of $R$ and $n \geq 1$. Since $R / I$ is a retractable ring, by the first part, $M I^{i} / M I^{i+1}$ are divisible $R / I$-modules for $i=0,1, \ldots, n$ with $I^{0}=R$. It follows that $M c=M$ for every $c \in R$ which is right regular modulo $I$.

(ii) By $[\mathbf{2 3}, 14.9], M_{R}$ has a factor $L$ such that $\operatorname{Soc}(L) \neq 0$. Thus by the retractable condition on $L$, we can deduce that $M$ has a maximal submodule, proving that $\mathrm{J}(M) \neq M$.

(iii) Let $0 \neq f \in \operatorname{Hom}_{R}(M, N)$. Then $f$ induces

$$
\bar{f}: M / \mathrm{J}(M) \rightarrow f(M) / f(\mathrm{~J}(M)) .
$$

Thus $f(\mathrm{~J}(M)) \subseteq \mathrm{J}(f(M)) \neq f(M)$ by (ii). Hence, by hypothesis, there exists a simple submodule $S$ of $M / \mathrm{J}(M)$ such that $S$ embeds in $N / f(\mathrm{~J}(M))$. Now by retractable condition on $N / f(\mathrm{~J}(M)), S \simeq N / K$ for some maximal submodule $K$ of $N$. It follows that

$$
\operatorname{Hom}_{R}(N / \mathrm{J}(N), M / \mathrm{J}(M)) \neq 0 .
$$

(iv) First note that if $M_{R}$ is nonsingular, then by hypothesis there is a nonzero map $f: \mathrm{E}(M) \rightarrow M$. Thus Ker $f$ is an essentially closed, and 
hence a direct summand of the injective $R$-module $\mathrm{E}(M)$. It follows that $\operatorname{Im} f$ is a nonzero injective submodule of $M_{R}$. Therefore, we can deduce that every nonzero nonsingular $R$-module contains a nonzero injective $R$-module. On the other hand, if $m$ is any nonzero element

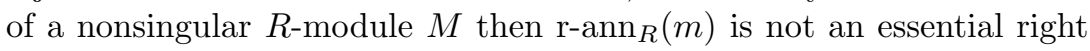
ideal of $R$, and so there exists a right ideal $A$ in $R$ such that $m A \simeq$ $A$. Consequently, if $M_{R}$ is nonsingular, then every nonzero submodule of $M_{R}$ contains a nonzero injective projective submodule. The proof is now completed by the fact that nonzero projective modules are not singular. To see this let $P_{R} \neq 0, P \oplus K=F$ and $F_{R}$ be free with basis $\left\{e_{i} \mid i \in I\right\}$. If $P \simeq F / K$ is singular then for every $i \in I$, there exists an essential right ideal $A_{i}$ of $R$ such that $e_{i} A_{i} \subseteq K$. Now $N:=\oplus_{i \in I} e_{i} A_{i}$ is an essential submodule of $F_{R}$ with $N \cap P=0$, contradiction. Therefore, $P_{R}$ is not singular.

(v) Let $M_{R}$ be nonsingular. If $0 \neq m \in \mathrm{J}(M)$, then by (iv) $m R$ contains a nonzero direct summand of $M_{R}$, but $m R$ is a small submodule of $M_{R}$, a contradiction. Thus $\mathrm{J}(M)=0$.

The following result together with Examples 3.9 show that the class of retractable rings properly lies between two classes of known rings. We first recall the necessary definitions. Following [7], a ring $R$ is called right $C P F$ if for all proper ideals $I$ of $R$, any faithful $R / I$-module is a generator in Mod- $R / I$. Artinian principal ideal rings are known to be CPF $[\mathbf{2 3}, 56.9(\mathrm{c})]$. Also in $[\mathbf{6}]$, the ring $R$ is called right HP (HiranoPark) if for every non-zero $R$-module $M$, the converse of Schur's Lemma holds (i.e., if $\operatorname{End}_{R}(M)$ is a division ring, then $M_{R}$ is a simple module). More recent works on HP rings are cited in the references. Rings over which any non-zero module has a maximal submodule are called right max rings; see [20] for an excellent reference on the subject.

Proposition 2.4. $\quad$ (i) Right CPF rings are retractable.

(ii) Any retractable rings is a right divisible, right max and $H P$ ring.

Proof: Part (i) follows from the definitions. For part (ii), note that $R$ is a right max ring by Proposition 2.3(ii). Now if $\operatorname{End}_{R}(M)$ is a division ring and $0 \neq N \leq M_{R}$, then the existence of a nonzero map $M_{R} \rightarrow N_{R}$ implies that $N=M$. Thus $M_{R}$ is simple and $R$ is an HP ring. Applying Proposition 2.3(i) for $M=R$ and $I=0$, we have that $R_{R}$ is divisible.

Lemma 2.5. Being (essentially) retractable is a Morita invariant property. 
Proof: Just note that in the definition of the (essentially) retractable modules, only categorical terms are used; see [2, Proposition 21.6].

Proposition 2.6. The class of retractable rings is closed under homomorphic image, Morita equivalence and finite product.

Proof: Let $\mathcal{C}$ be the class of all retractable rings. Clearly, $\mathcal{C}$ is closed under homomorphic image and Morita equivalence by Lemma 2.5. Now suppose $R_{1}$ and $R_{2}$ are retractable rings and set $T=R_{1} \oplus R_{2}$. If $M$ is a $T$-module then $M=M e_{1} \oplus M e_{2}$ where $e_{1}$ and $e_{2}$ are central orthogonal idempotents in $T$ such that $e_{1} R_{2}=e_{2} R_{1}=0$ and $e_{1}+$ $e_{2}=1_{T}$. Clearly $M e_{i}$ is naturally an $R_{i}$-module for $i=1,2$. Now let $0 \neq m \in M$. We have $m=m 1_{T}=m e_{1}+m e_{2}$. Hence there is $i \in$ $\{1,2\}$ such that $m e_{i} \neq 0$. So by our assumption, there exists a nonzero $R_{i}$-homomorphism $f_{i}: M e_{i} \rightarrow m R_{i} e_{i}$. Since $e_{1} R_{2}=e_{2} R_{1}=0, M e_{i}$ is a $T$-submodule of $M$ and $f_{i}$ is a $T$-module homomorphism. Now $f_{i} \pi_{i}$ is a nonzero $T$-module homomorphism from $M$ to $m T$ where $\pi_{i}: M \rightarrow M e_{i}$ is the natural projection. Hence $M_{T}$ is retractable, and $T$ is a retractable ring.

In the following we investigate the retractability of the class $\sigma\left[M_{R}\right]$ when $M_{R}$ is a locally Noetherian module. Recall from $[\mathbf{2 3}, 15]$ that $\sigma\left[M_{R}\right]$ is a full subcategory of the category Mod- $R$ whose objects are submodules of modules which are generated by $M_{R}$. Also a module $M_{R}$ is said to be polyform if $\operatorname{Hom}_{R}(M / N, \hat{M})=0$ for any $N \leq_{e} M_{R}$. Here $\hat{M}$ is the $M$-injective envelope of $M_{R}$ in $\sigma\left[M_{R}\right]$. Alternatively, $M_{R}$ is polyform if and only if $\operatorname{End}_{R}(\hat{M})$ is a regular ring [5, 4.9]. The class of polyform modules properly contains both the class of nonsingular and the class of semisimple modules. It is known that any submodule and any quasi-injective hull of a polyform module is again polyform.

Proposition 2.7. Suppose that $M_{R}$ is polyform such that nonzero direct summands of $\hat{M}$ are retractable $R$-modules. If $M_{R}$ is locally Noetherian or it has acc (dcc) on direct summands, then $M_{R}$ is semisimple.

Proof: The first we show that every indecomposable submodule of $M_{R}$ is a simple $M$-injective $R$-module. Let $U$ be an indecomposable submodule of $M_{R}$ and $0 \neq K \leq U$. Then $\hat{U}$, the $M$-injective hull of $U$, is a direct summand of $\hat{M}$, and so by our assumption $\hat{U}$ is retractable. Now similar to the proof of Proposition 2.3(iv), $U$ contains a nonzero $M$-injective submodule of $K$. Therefore, $K=U$ by the indecomposable condition on $U$, as desired. Now if $M_{R}$ has acc (dcc) on direct summands, then we are done by $\left[\mathbf{2}\right.$, Proposition 10.14]. Let $M_{R}$ be a local noetherian. 
By the first part and [5, Corollary 5.2(2)], we can deduce $\operatorname{Soc}(M)$ is an essential submodule of $M$. On the other hand, $\operatorname{Soc}(M)$ is an $M$-injective submodule of $M$ by $[\mathbf{5}, 2.5(\mathrm{c})]$. It follows that $\operatorname{Soc}(M)=M$.

Corollary 2.8. Over a right Noetherian ring $R$, a nonzero module $M_{R}$ is semisimple if and only if it is polyform and the class $\sigma\left[M_{R}\right]$ is retractable.

Proof: By Proposition 2.7.

By Proposition 2.4 and the next lemma, we observe that if $R$ is retractable, then every Artinian module is Noetherian. The converse will be investigated in Proposition 2.10.

Lemma 2.9. Let $M$ be a nonzero $R$-module and $R$ is a right max ring. If every factor module of $M$ has finite uniform dimension, then $M_{R}$ is Noetherian.

Proof: Just note that if $N \leq M_{R}$ is not finitely generated then by $[\mathbf{5}$, 5.11], there exists a finitely generated submodule $K \leq N$ such that $N / K$ has no maximal submodule, a contradiction.

Proposition 2.10. Over a retractable ring, a module is Artinian if and only if it is Noetherian and its second singular submodule is Artinian.

Proof: The necessity follows from Proposition 2.4(ii) and Lemma 2.9. Let $M_{R}$ be noetherian and $\mathrm{Z}_{2}(M)$ be Artinian. Note that $L:=M / \mathrm{Z}_{2}(M)$ is nonsingular and hence a polyform module. Now apply Proposition 2.7 for the module $L$ to deduce that $L_{R}$ is a semisimple noetherian module. Since now $L$ and $\mathrm{Z}_{2}(M)$ are Artinian, $M_{R}$ is Artinian.

\section{Characterization of some classes of rings}

In this section, we give new characterizations for semisimple Artinian rings and certain semi-Artinian rings in terms of retractable rings. Two important subclasses of the class of Artinian rings are the class of Artinian principal ideal rings and the class of rings over which every right (and left) module is a direct sum of cyclic modules (Köthe rings). Let $\mathcal{K}($ resp. $\mathcal{A P})$ be the classes of Köthe (resp. Artinian principal ideal) rings. In [13], it is proved that $\mathcal{A P} \subseteq \mathcal{K}$ and it is asked what the Köthe rings are; see also [17, Appendix B, Problem 2.48]. Recently, in [4, Theorem 3.1], it is proved that normal Köthe rings are Artinian principal ideal rings. A restatement of our Corollary 3.8, gives $\mathcal{A P} \subseteq \mathcal{R}$ where $\mathcal{R}$ is the class of retractable rings. Hence, if a Köthe ring is an Artinian 
principal ideal ring then it must be a retractable ring. We first characterize when a (semi-)Artinian ring is retractable and then determine when Köthe rings are Artinian principal ideal rings.

Recall from $[\mathbf{1 4}, 11.9]$ a ring $R$ is said to be right Goldie if $R$ has ascending chain condition on right annihilators and the uniform dimension of $R_{R}$ is finite. Left Goldie rings are defined similarly. Semiprime right Goldie rings are known to be right nonsingular. In [11, Proposition 11], it is shown that right nonsingular HP rings with finite uniform dimension are semisimple Artinian. Hence, by Proposition 2.4, retractable semiprime right Goldie rings are precisely semisimple Artinian rings. In the following, we obtain a similar result for retractable semiprime left (right) Goldie rings.

Proposition 3.1. $\quad$ (i) Retractable domains are precisely division rings.

(ii) The ring $R$ is a semiprime left (right) Goldie retractable ring if and only if $R$ is a semisimple Artinian ring if and only if $R$ is a right nonsingular retractable ring with acc (dcc) on direct summand right ideals.

Proof: (i) This follows from Proposition 2.3(i).

(ii) Suppose that $R$ is semiprime left Goldie and let $I$ be an essential left ideal of $R$. Then $I$ contains a regular element $x$ and so $R x=R$, by Proposition 2.3(i). It follows that ${ }_{R} R$ has no proper essential left ideals, proving that $R$ is a semisimple Artinian ring. The second equivalence is obtained by Proposition 2.7.

A ring $R$ is said to be reduced if $R$ has no nonzero nilpotent elements. A reduced ring which is a regular ring is called strongly regular; see [23, 3.11] for more information. A ring $R$ is said to be right (left) semiArtinian if every nonzero right (left) $R$-module has a nonzero socle, and $R$ is called semi-Artinian if it is right and left semi-Artinian. In $[\mathbf{8}$, Theorem 2.7], it is shown that for a commutative ring, the semi-Artinian condition implies the retractable condition. The converse follows by $[\mathbf{1 5}$, Theorem 8]. We will give a generalization of this result in Theorem 3.4.

Theorem 3.2. A ring is reduced and retractable if and only if it is a (right) semi-Artinian strongly regular ring.

Proof: For the sufficiency, note that since $R$ is strongly regular, $R$ is reduced, right ideals in $R$ are two sided and cyclic $R$-modules are flat. Hence, all simple $R$-modules are injective by [14, Corollary 3.6A]. It follows that the semi-Artinian ring $R$ is retractable. Conversely, let $R$ be a reduced retractable ring, $0 \neq a \in R, M=a R$, and $I=\mathrm{r}_{-} \operatorname{ann}_{R}(a)$. 
Since $R$ is a reduced ring, $I$ is an ideal of $R$ and so we have $I \cap a R=$ 0 . It follows that $a$ is right regular modulo $I$. Hence $M a=M$ by Proposition 2.3, proving that $R$ is a regular ring. Now $R$ is strongly regular by the reduced condition on $R$.

To show that $R$ is semi-Artinian, we will show that every cyclic $R$-module contains an injective $R$-module $[\mathbf{5}, 15.11]$. Suppose now $B \leq$ $R_{R}$. Since $R$ is a strongly regular ring, $B$ is an ideal of $R$ and the ring $R / B$ is (right) nonsingular. By hypothesis, $R / B$ is also a retractable ring and so it contains an injective $R / B$-module by Proposition 2.3(iv). On the other hand, $R / B$ is a flat left $R$-module and so by [14, Corollary 3.6(A)], every injective right $R / B$-module is injective as a right $R$-module, as desired.

Following [2, p. 314], a non-empty subset $Y$ of $R$ is called left T-nilpotent provided for each sequence $y_{1}, y_{2}, y_{3}, \ldots$ of elements of $Y$ there exists a positive integer $n$ such that $y_{1} y_{2} \ldots y_{n}=0$.

Proposition 3.3. Let $R$ be a ring with $\mathrm{J}(R) \subseteq \operatorname{Cent}(R)$. Then $R$ is a retractable ring if and only if $R / \mathrm{J}(R)$ is a retractable ring and $\mathrm{J}(R)$ is a T-nilpotent ideal.

Proof: $(\Rightarrow)$ This follows from Proposition 2.4 and [2, Remark 28.5].

$(\Leftarrow)$ Let $M_{R}$ be a nonzero $R$-module and $J=\mathrm{J}(R)$. By Theorem 2.2, we shall show that $M_{R}$ is essentially retractable. If $M J=0$ then $M$ is an $R / J$-module and we are done. If $M J \neq 0$, then there exists $r \in$ $J$ such that $M r J=0$ but $M r \neq 0$. Since $J \subseteq \operatorname{Cent}(R), M r$ is an $R / J$-module and so it is essentially retractable as an $R / J$-module as well as $R$-module. Now multiplication by $r$ defines a nonzero homomorphism $f$ in $\operatorname{End}_{R}(M)$ such that $f(M)=M r$. Thus $M_{R}$ is essentially retractable by Lemma 2.1 .

A ring $R$ is called normal if all idempotent elements in $R$ are central.

Theorem 3.4. If $R$ is a retractable ring such that $R / \mathrm{J}(R)$ is reduced then $R$ is a left semi-Artinian ring and $R / \mathrm{J}(R)$ is a right semi-Artinian normal ring. The converse holds if $\mathrm{J}(R) \subseteq \operatorname{Cent}(R)$.

Proof: Let $R$ be a retractable ring and $R / \mathrm{J}(R)$ be reduced. By Theorem 3.2, $R / \mathrm{J}(R)$ is a (right) semi-Artinian strongly regular ring. By Proposition 2.4, $R$ is a right max ring and so $\mathrm{J}(R)$ is a right T-nilpotent. It follows that $R$ is left semi-Artinian [20, Lemma 2.12]. The last statement is true because reduced rings are normal. 
Suppose now that $J=\mathrm{J}(R) \subseteq \operatorname{Cent}(R), R$ is left semi-Artinian and $R / \mathrm{J}(R)$ is a right semi-Artinian normal ring. Because $R$ is left semiArtinian, $J$ is a T-nilpotent ideal [2, Remark 28.5(2)]. Thus by Proposition 3.3, it is enough to show that $R / J$ is a retractable ring. Since $R / J$ is semi-Artinian normal ring with zero Jacobson radical, it is a regular ring by $[\mathbf{1}$, Corollary 1.4]. Hence $R / J$ is a strongly regular ring by our assumption. The proof is now completed by Theorem 3.2.

Corollary 3.5. Suppose that $R$ is a ring Morita equivalent to a ring $S$ such that $S / \mathrm{J}(S)$ is a reduced ring and $\mathrm{J}(S) \subseteq \operatorname{Cent}(S)$. Then $R$ is a retractable ring if and only if $R$ is a semi-Artinian ring.

Proof: By Theorem 3.4, the ring $S$ is retractable if and only if it is semiArtinian. Hence, the proof is completed by Proposition 2.6 and the fact that being semi-Artinian is a Morita invariant property.

Following [5, Section 6], the Krull dimension of a module $M_{R}$ is denoted by $\mathrm{K}-\operatorname{dim}\left(M_{R}\right)$ and $\mathrm{K}-\operatorname{dim}\left(R_{R}\right)$ is called the right Krull dimension of $R$. Clearly, K-dim $\left(M_{R}\right) \leq 0$ if and only if $M_{R}$ is Artinian. Noetherian modules have Krull dimension and modules with Krull dimension are known to have finite uniform dimension [5, 6.2].

Theorem 3.6. The following statements are equivalent for a ring $R$.

(i) $R$ is a retractable ring and every cyclic $R$-module has finite uniform dimension.

(ii) $R$ is a right Noetherian retractable ring.

(iii) $R$ is a retractable ring with right Krull dimension.

(iv) $R$ is a finite product of matrix rings over right Artinian local rings.

(v) $R$ is a right Artinian for which the product of any two prime ideal commutes.

(vi) $R$ is a right Artinian retractable ring.

Proof: (i) $\Rightarrow$ (ii). This follows from Proposition 2.4 and Lemma 2.9.

(ii) $\Rightarrow$ (iii). This is obtained by $[\mathbf{5}, 6.2]$.

(iii) $\Rightarrow$ (iv). By a well known result $\mathrm{K}-\operatorname{dim}\left(R_{R}\right)=\operatorname{Sup}\{\operatorname{right} \mathrm{K}-\operatorname{dim}(R / P) \mid$ $P$ is a prime ideal of $R$ \}. Also by [5, Theorem 6.6], $R / P$ is a prime right Goldie ring and so it is Artinian by Proposition 3.1. Thus $R$ is a right Artinian ring. By [2, Proposition 10.17], we may suppose that $R$ is indecomposable as a ring. Thus $R_{R}$ has no non-trivial fully invariant direct summand. On the other hand, by [2, Proposition 28.13], $R_{R} \simeq$ $e_{1} R^{\left(A_{1}\right)} \oplus \cdots \oplus e_{n} R^{\left(A_{n}\right)}$ for some $n \geq 1$ where each $e_{i} R e_{i}$ is a local ring. Now the indecomposable condition on $R$ with an application of 
Proposition 2.3(iii) for $M=e_{i} R$ and $N=e_{j} R(i \neq j)$ imply that $R_{R} \simeq e_{i} R^{\left(A_{i}\right)}$, hence $R \simeq \mathrm{M}_{A_{i}}\left(e_{i} R e_{i}\right)$ for some $i$. The proof is complete.

(iv) $\Rightarrow(\mathrm{v})$. This follows by the fact that in any right Artinian local ring the Jacobson radical is the unique prime ideal.

(v) $\Rightarrow$ (vi). Suppose that every two prime ideal in $R$ commute together, $M$ is a nonzero $R$-module and $S$ is a simple submodule of $M$ with $P_{1}:=$ $\operatorname{ann}_{R}(S)$. We shall show that $\operatorname{Hom}_{R}(M, S)$ is nonzero. Let $J=\mathrm{J}(R)$, since $R$ is right Artinian, $J^{k}=0$ for some $k \geq 1$. We claim that $M P_{1} \neq$ $M$. If $M P_{1}=M$, then $R$ is not a local ring (otherwise, $P_{1}=J$ is a nilpotent ideal and so $\left.M P_{1} \neq M\right)$. Therefore, suppose that $P_{1}, \ldots, P_{n}$ $(n \geq 2)$ are all distinct prime (maximal) ideals in $R$. Then we have $S\left(P_{2} \ldots P_{n}\right)^{k} \subseteq M\left(P_{2} \ldots P_{n}\right)^{k}=M P_{1}^{k}\left(P_{2} \ldots P_{n}\right)^{k} \subseteq M J^{k}=0$. It follows that $P_{i} \subseteq P_{1}$ for some $i \geq 2$, a contradiction. Therefore $M P_{1} \neq$ $M$, as claimed. Now $M / M P_{1}$ is a nonzero module over the semisimple ring $R / P_{1}$ and so there exists a nonzero homomorphism from $M / M P_{1}$ to the (unique) simple $R / P_{1}$-module $S$. This shows that $\operatorname{Hom}_{R}(M, S)$ is nonzero.

(vi) $\Rightarrow$ (i). This is well known.

An $R$-module $M$ is called finitely annihilated provided there exist a positive integer $n$ and elements $m_{i} \in M(1 \leq i \leq n)$ such that $A:=\operatorname{ann}_{R}(M)=\cap_{i} \operatorname{ann}_{R}\left(m_{i}\right)$, equivalently there exists an embedding $\theta: R / A \rightarrow M^{(n)}$. It is well known that a ring $R$ is right Artinian if and only if every right $R$-module is finitely annihilated. Hence, if the ring $R / A$ is right Artinian, then $M_{R}$ is finitely annihilated.

Corollary 3.7. Let $R$ be a retractable ring. Then a nonzero $R$-module $M$ is finitely annihilated with finite uniform dimensional factors if and only if $M_{R}$ is finitely generated and the ring $R / \operatorname{ann}_{R}(M)$ is a finite product of matrix rings over right Artinian local rings.

Proof: The sufficiency is clear. Conversely, by Proposition 2.4 and Lemma 2.9, $M_{R}$ is Noetherian. Hence $R / \operatorname{ann}_{R}(M)$ is a right Noetherian ring by our assumption. The proof is now completed by Theorem 3.6.

Corollary 3.8. Right Artinian principal right ideal rings are retractable.

Proof: By $[\mathbf{2 3}, 56.3]$ and Theorem 3.6.

Examples 3.9. (i) If $R$ is any ring and $e$ is an idempotent element of $R$ such that $e R \cap \mathrm{l}-\operatorname{ann}_{R}(e)$ contains a nonzero right ideal $I$ then $e R$ is not retractable as an $R$-module and consequently $R$ is not a retractable 
ring. To see this let $0 \neq f \in \operatorname{Hom}_{R}(e R, I)$, then $f(e R)=f(e) e R=0$, a contradiction.

(ii) Suppose that $A$ and $B$ are rings and ${ }_{A} M_{B}$ is a nonzero bimodule. Let $R=\left[\begin{array}{cc}A & M \\ 0 & B\end{array}\right]$, and $e=\left[\begin{array}{ll}1 & 0 \\ 0 & 0\end{array}\right]$, then $\left[\begin{array}{cc}0 & M \\ 0 & 0\end{array}\right]$ lies in $e R \cap l-a_{n}(e)$. So by (i), $R$ is not a retractable ring. Thus one may easily produce semi-Artinian rings which are not retractable.

(iii) For any ring $R$, the ring $R[x]$ is never retractable (Proposition 2.4(ii)).

(iv) There exists a retractable ring which is not CPF. Suppose that $S=\mathbb{Q}\left[x_{i} \mid i \in \mathbb{N}\right]$ and $I$ is the ideal of $S$ generated by the subset $\left\{x_{i} x_{j}, x_{k}^{k+1} \mid i \neq j, k \in \mathbb{N}\right\}$ and $R=S / I$. Then it is easy to verify that $R$ is a local ring with $\mathrm{J}(R)=J=\left\langle\bar{x}_{i} \mid i \in \mathbb{N}\right\rangle$. In view of Proposition 3.3, to show that $R$ is retractable, we shall show that $J$ is T-nilpotent. Let $f_{i} \in J$ and $f_{1} \in A:=\left\langle\bar{x}_{1}, \ldots, \bar{x}_{n}\right\rangle$. Thus $f_{1} f_{2} \ldots f_{n+1} \in A J^{n}=0$. To proof that $R$ is not a CPF ring, consider the faithful $R$-module $M=$ $\bigoplus_{i} R /\left(\oplus_{j \neq i} \bar{x}_{j} R\right)$. If $M_{R}$ is generator, then $R$ must be embedded in $M_{R}^{(k)}$ for some $k \geq 1$, but every element in $M^{(k)}$ has nonzero annihilator, a contradiction. Hence $M_{R}$ is not generator, and so $R$ is not a CPF ring.

(v) There exists a divisible, HP, max ring which is not retractable. Let $R=\mathbb{Q}^{\mathbb{N}}$ be the countable product of $\mathbb{Q}$ and $I=\mathbb{Q}^{(\mathbb{N})}$. Then it is well known that $R$ is a self-injective regular $\operatorname{ring}$ such that $\operatorname{Soc}(R / I)_{R}=0$. Thus $R$ is a divisible, max ring and it is an HP ring by [11, Corollary 15], but $R$ is not a retractable ring by Corollary 3.5.

A characterization of Artinian principal ideal rings in [23, 56.9], shows that a ring $R$ is Artinian principal ideal ring if and only if such is $\operatorname{Mat}_{n}(R)$. If $S$ is a ring, we say that $S$ is a matrix ring direct summand (matrix rds) of $R$ whenever $\operatorname{Mat}_{n}(S) \times T \simeq R$ for some $\operatorname{ring} T$ and $n \geq 1$.

Theorem 3.10. Let $R$ be a Köthe ring. Then $R$ is an Artinian principal ideal ring if and only if $R$ is a retractable ring and every local ring which is a matrix rds of $R$ is a Köthe ring.

Proof: $(\Rightarrow)$ By Corollary $3.8 R$ is a retractable ring. Suppose that $S$ is a matrix rds of $R$. By hypothesis and [23, 56.9], $S$ is an Artinian principal ideal ring. Hence $S$ is a Köthe ring by [13].

$(\Leftarrow)$ Since $R$ is a Köthe ring, it is Artinian. Hence, by Theorem 3.6, $R \simeq \bigoplus_{i} \operatorname{Mat}_{n_{i}}\left(R_{i}\right)$ such that each $R_{i}$ is local and a matrix rds of $R$. Thus by hypothesis, each $R_{i}$ is a local Köthe ring. The proof is completed by $[4$, Theorem 3.1]. 
Corollary 3.11. The following statements are equivalent.

(i) $\mathcal{K}=\mathcal{A P}$.

(ii) $\mathcal{K} \subseteq \mathcal{R}$ and for any local ring $R$, if $\operatorname{Mat}_{n}(R) \in \mathcal{K}$ for some $n \geq 1$, then $R \in \mathcal{K}$.

Proof: This follows from Theorem 3.10.

Now we shall consider when a Köthe ring is a retractable ring. First we state a lemma; note that the equivalence (i) $\Leftrightarrow$ (ii) of below was obtained in [8, Proposition 2.2].

Lemma 3.12. Let $I$ be a proper right ideal in a ring $R$. Then the following statements are equivalent.

(i) The cyclic $R$-module $R / I$ is retractable.

(ii) For any right ideal $J$, either $J \subseteq I$ or there exists $x \in J \backslash I$ such that $x I \subseteq I$.

(iii) For each $x \in R \backslash I$, there exists $r \in R$ such that $x r \notin I$ and $x r I \subseteq I$.

Proof: (i) $\Rightarrow$ (ii). If $J \subseteq I$, then we are through. Hence, let $a \in J \backslash I$, then by (i), there exists a nonzero homomorphism $f: R / I \rightarrow(a R+I) / I$ with $f(1+I)=a r+I$ for some $r \in R$. We now have $x I \subseteq I$, where $x=\operatorname{ar} \in J \backslash I$.

(ii) $\Rightarrow$ (iii). Suppose that $x \in R \backslash I$. Since the right ideal $J=x R+I$ properly contains $I$, by (ii) there exists $y \in J \backslash I$ such that $y I \subseteq I$. Write $y=x r+i$ for some $r \in R$ and $i \in I$. Then $x r \notin I$ as $y \notin I$, and $x r I \subseteq I$ because $y I \subseteq I$.

(iii) $\Rightarrow$ (i). Suppose $I<J \leq R_{R}$. Pick $j \in J \backslash I$, then by (iii) there exists $r \in R$ with $j r \notin I$ and $j r I \subseteq I$. Let $x=j r$ then the left multiplication by $x$ yields a nonzero homomorphism from $R / I$ to $J / I$.

A module $M_{R}$ is called co-cyclic if it has a simple essential submodule.

Theorem 3.13. Let $R$ be a right Köthe ring. The following statements are equivalent.

(i) The ring $R$ is retractable.

(ii) $M \neq M P$ for any nonzero cyclic co-cyclic $R$-module $M$ with $P \in$ $\operatorname{Ass}(M)$.

(iii) For any right ideal $I \leq R$ such that $(R / I)_{R}$ is co-cyclic and every $x \in R \backslash I$, there exists $r \in R$ such that $x r \notin I$ and $x r I \subseteq I$. 
Proof: Note that if $Y_{R}$ is retractable, then for any module $X_{R}$, the $R$-module $Y \oplus X$ is always essentially retractable by Lemma 2.1. Therefore, since $R$ is Köthe, by Theorem 2.2, we see that $R$ is retractable if and only if nonzero cyclic $R$-modules are retractable. Let $M_{R}$ be a cyclic $R$-module. Since $R$ is right Artinian, $M$ is retractable if and only if $\operatorname{Hom}_{R}(M, S) \neq 0$ for any simple submodule $S \leq M_{R}$. If $S$ is not an essential submodule of $M$, it can be essentially embedded in some factor of $M$. Thus we can conclude that every nonzero cyclic $R$-modules is retractable if and only if every nonzero cyclic co-cyclic $R$-modules is retractable. Hence, (i) $\Leftrightarrow$ (iii) by Lemma 3.12. For (i) $\Leftrightarrow$ (ii) note that if $M$ is a nonzero cyclic co-cyclic $R$-module and $P \in \operatorname{Ass}(M)$. Then $P=\operatorname{ann}_{R}(S)$ where $S$ is the unique simple submodule of $M$. Since now $R / P$ is a simple Artinian ring, we have $\operatorname{Hom}_{R}(M, S) \neq 0$ if and only if $\operatorname{Hom}_{R}(M, R / P) \neq 0$ if and only if $M \neq M P$. The proof is complete.

Acknowledgements. The authors are grateful to the referee for valuable suggestions towards improvement of the presentation. They also wish to express their gratitude to Professors A. Haghany and O. A. S. Karamzadeh for communications regarding the subject matter of this paper and thank Professor M. Behboodi for bringing [4] to their attention.

\section{References}

[1] A. N. Abyzov, Weakly regular modules over normal rings, (Russian), Sibirsk. Mat. Zh. 49(4) (2008), 721-738; translation in: Sib. Math. J. 49(4) (2008), 575-586. DOI: 10.1007/s11202-008-0055-3.

[2] F. W. Anderson And K. R. Fuller, "Rings and categories of modules", Second edition, Graduate Texts in Mathematics 13, Springer-Verlag, New York, 1992. DOI : 10.1007/978-1-4612-4418-9.

[3] J. A. BeACHY, $M$-injective modules and prime $M$-ideals, Comm. Algebra 30(10) (2002), 4649-4676. DOI : 10.1081/AGB-120014660.

[4] M. Behboodi, A. Ghorbani, S. Moradzadeh-Dehkordi, And S. H. Shojaee, On left Köthe rings and a generalization of the Köthe-Cohen-Kaplansky theorem, Proc. Amed Math. Soc. (to appear).

[5] N. V. Dung, D. V. Huynh, P. F. Smith, and R. Wisbauer, "Extending modules", Pitman Research Notes in Mathematics Series 313, Longman Scientific \& Technical, Harlow; copublished in the United States with John Wiley \& Sons, Inc., New York, 1994. 
[6] C. Faith, Indecomposable injective modules and a theorem of Kaplansky, Comm. Algebra 30(12) (2002), 5875-5889. DOI: 10.1081/ AGB-120016019.

[7] C. Faith and S. Page, "FPF ring theory. Faithful modules and generators of mod- $R$ ", London Mathematical Society Lecture Note Series 88, Cambridge University Press, Cambridge, 1984.

[8] A. Haghany, O. A. S. Karamzadeh, and M. R. Vedadi, Rings with all finitely generated modules retractable, Bull. Iranian Math. Soc. 35(2) (2009), 37-45, 270.

[9] A. Haghany and M. R. Vedadi, Study of semi-projective retractable modules, Algebra Colloq. 14(3) (2007), 489-496.

[10] A. Haily and M. Alaoui, Perfect rings for which the converse of Schur's lemma holds, Publ. Mat. 45(1) (2001), 219-222. DOI: 10.5565/PUBLMAT_45101_10.

[11] Y. Hirano AND J. K. PARK, Rings for which the converse of Schur's lemma holds, Math. J. Okayama Univ. 33 (1991), 121-131.

[12] S. M. KHURI, The endomorphism ring of a nonsingular retractable module, East-West J. Math. 2(2) (2000), 161-170.

[13] G. Kӧтне, Verallgemeinerte Abelsche Gruppen mit hyperkomplexem Operatorenring, Math. Z. 39(1) (1935), 31-44. DOI: 10.1007/BF01201343.

[14] T. Y. LAM, "Lectures on modules and rings", Graduate Texts in Mathematics 189, Springer-Verlag, New York, 1999. DOI: 10.1007/ 978-1-4612-0525-8.

[15] K. Ohtake, Commutative rings over which all torsion theories are hereditary, Comm. Algebra 9(15) (1981), 1533-1540. DOI: 10.1080/00927878108822663.

[16] S. T. Rizvi and C. S. Roman, On direct sums of Baer modules, J. Algebra 321(2) (2009), 682-696. DOI : 10.1016/j.jalgebra. 2008. 10.002 .

[17] L. Sabinin, L. Sbitneva, and I. Shestakov, eds., "Nonassociative algebra and its applications", Proceedings of the 5th International Conference held in Oaxtepec, July 27-August 2, 2003, Lecture Notes in Pure and Applied Mathematics 246, Chapman \& Hall/CRC, Boca Raton, FL, 2006. DOI: 10.1201/9781420003451.

[18] P. F. Smith, Modules with many homomorphisms, J. Pure Appl. Algebra 197(1-3) (2005), 305-321. DOI: 10.1016/j.jpaa.2004.09. 001.

[19] P. F. Smith and M. R. Vedadi, Submodules of direct sums of compressible modules, Comm. Algebra 36(8) (2008), 3042-3049. DOI : $10.1080 / 00927870802110854$. 
[20] A. A. Tuganbaev, Rings whose nonzero modules have maximal submodules, J. Math. Sci. (New York) 109(3) (2002), 1589-1640. DOI: $10.1023 / \mathrm{A}: 1013981125581$.

[21] D. K. TÜtüncü, N. Orhan Ertaş, R. Tribak, and P. F. Smith, On fully idempotent modules, Comm. Algebra 39(8) (2011), 2707-2722. DOI: 10.1080/00927872.2010.489916.

[22] M. R. Vedadi, Essentially retractable modules, J. Sci. Islam. Repub. Iran 18(4) (2007), 355-360.

[23] R. WisBauer, Foundations of module and ring theory, Revised and translated from the 1988 German edition, Algebra, Logic and Applications 3, Gordon and Breach Science Publishers, Philadelphia, PA, 1991.

[24] J. M. Zelmanowitz, Correspondences of closed submodules, Proc. Amer. Math. Soc. 124(10) (1996), 2955-2960. DOI: 10.1090/S00029939-96-03469-7.

Department of Mathematical Sciences

Isfahan University of Technology

Isfahan, 84156-83111

Iran

E-mail address: y.toloei@math.iut.ac.ir

E-mail address: mrvedadi@cc.iut.ac.ir

Primera versió rebuda el 20 de gener de 2012, darrera versió rebuda el 29 de juny de 2012. 